\title{
Silencing of the $A B C C 4$ gene by RNA interference reverses multidrug resistance in human gastric cancer
}

\author{
GUANGYU ZHANG $^{1 *}$, ZHENRAN WANG $^{1 *}$, FENG QIAN $^{2}$, CHEN ZHAO $^{1}$ and CHAOWEN SUN ${ }^{1}$ \\ ${ }^{1}$ Department of Gastrointestinal Surgery, Affiliated Hospital of Guilin Medical University, Guilin 541001; \\ ${ }^{2}$ Department of General Surgery, The Southwest Hospital of the Third Military \\ Medical University, Shapingba, Chongqing 400038, P.R. China
}

Received September 1, 2014; Accepted November 24, 2014

DOI: $10.3892 /$ or.2014.3702

\begin{abstract}
The identification of genes associated with recurrent drug resistance in gastric cancer and the elucidation of the underlying molecular mechanisms associated with recurrent drug resistance in gastric cancer are important for the effective treatment and prognosis of this cancer. Variations in the expression level of the ATP-binding cassette subfamily $\mathrm{C}$ member 4 (ABCC4) gene are correlated with the recurrence, development and chemotherapeutic susceptibility of various types of cancers. In the present study, we demonstrated that the $A B C C 4$ gene was highly expressed in multiple types of gastric cancer cells, and $A B C C 4$ expression was even more prominent in the drug-resistant gastric cancer cells. Conversely, in normal gastric mucosal cells, $A B C C 4$ expression was very low or undetectable. We used RNA interference to decrease the expression of $A B C C 4$ in drug-resistant gastric cancer cells, which resulted in an increase in apoptosis and cell cycle arrest in the $\mathrm{G} 1$ phase. In addition, we found that $A B C C 4$ knockdown in 5-fluorouracil (5-FU)-resistant cancer cells restored 5-FU sensitivity, resulting in the inhibition of cell proliferation and tumour growth in nude mice. Our results showed that inhibition of $A B C C 4$ gene expression can inhibit the proliferation of multidrug-resistant gastric cancer cells and can enhance gastric cancer cell sensitivity to chemotherapeutic drugs.
\end{abstract}

\section{Introduction}

Gastric cancer is one of the most common malignant tumours. Despite significant advances in the diagnosis and treatment of gastric cancer, the mortality associated with gastric

Correspondence to: Dr Guangyu Zhang, Department of Gastrointestinal Surgery, Affiliated Hospital of Guilin Medical University, 15 Lequn Road, Guilin 541001, P.R. China

E-mail: gygyzhang@163.com

${ }^{*}$ Contributed equally

Key words: ABCC4 gene, gastric cancer, RNA interference, multidrug resistance cancer accounts for over $10 \%$ of the total cancer mortalities worldwide and remains the second most common cause of cancer-related mortality after lung cancer $(1,2)$. At present, the most commonly used treatment methods for gastric cancer are surgery, radiotherapy and chemotherapy, and comprehensive treatment depending on the patient-specific disease manifestation. Of the available treatment methods for gastric cancer, chemotherapy represents an extremely important and irreplaceable option. However, since gastric cancer cells are prone to the development of multidrug-resistance phenotypes, many chemotherapeutic drugs are ineffective for gastric cancer.

With technological advances in molecular biology, biological treatments such as gene therapy have become areas of interest for the development of novel cancer therapeutics. The introduction of RNA interference (RNAi) technology offers a way to experimentally determine valid targets for gene therapy, as RNAi can be used to elucidate the mechanisms underlying the occurrence of multidrug-resistance and recurrence in gastric cancer.

Many molecular mechanisms associated with the generation of multiple-drug resistance in gastric cancer exist, of which the abnormal expression of the ATP-binding cassette subfamily $\mathrm{C}$ member 4 (ABCC4) protein plays a key role. $\mathrm{ABCC} 4$ protein, also known as the multi-drug resistanceassociated protein, is an important member of the ATP-binding cassette (ABC) transporter family. ABCC4 was first identified on the basal membrane of prostate epithelial cells (3). ABCC4 controls the redistribution and excretion of many anti-viral drugs, antibiotics, cytostatic drugs, and cardiovascular drugs and plays key roles in the protection of cells and cellular signal transduction pathways (4). In addition, ABCC4 can transport intracellular drugs outside of cells, which is often used by cancer cells as a drug resistance mechanism (5). Recent studies have indicated that ABCC4 expression is closely associated with the occurrence of malignant tumours and drug resistance. Studies have also confirmed that ABCC4 is overexpressed in gliomas, neuroblastomas, retinoblastomas and melanomas as well as colon cancer and colorectal cancer cells (6-9). In addition, ABCC4 expression is correlated with drug resistance in leukaemia and ovarian cancer cells $(10,11)$.

The relationship between $A B C C 4$ gene expression and multidrug-resistance in gastric cancer has not been reported. However, studies have shown that $A B C C 4$ gene expression 
is correlated with proliferation capacity and drug resistance in leukaemia, ovarian cancer and colon cancer cells (10-12). Therefore, we used RNAi to knock down $A B C C 4$ gene expression in multidrug-resistant gastric cancer cells to study the role of $A B C C 4$ gene expression in the occurrence and progression of multiple-drug-resistant gastric cancer.

\section{Materials and methods}

Cells, antibodies and reagents. Human gastric mucosal GES-1 cells, human gastric cancer MGC-803 cells, and multidrugresistant SGC-7901/Fu cells were purchased from American Type Culture Collection (ATCC; Rockefeller, MD, USA). ABCC4, P-gp, Bax, Bcl-2, CDK4 and cyclin D1 antibodies were purchased from Abcam (Santa Cruz, CA, USA). PI was purchased from Sigma (Hercules, CA, USA), siRNA was synthesised by GenePharma (Shanghai, China), and the RT-PCR reagent kit [Takara RNA PCR kit (AMV) version 3.0] was purchased from Takara (Dalian, China).

Cell culture. Human gastric mucosal GES-1 cells, human gastric cancer MGC-803 cells, and human gastric cancer SGC-7901 cells were cultured in RPMI-1640 culture medium (Carlsbad, CA, USA) containing $10 \%$ foetal bovine serum (FBS), $100 \mathrm{U} / \mathrm{ml}$ penicillin and $100 \mu \mathrm{g} / \mathrm{ml}$ streptomycin at $37^{\circ} \mathrm{C}$ in the presence of $5 \% \mathrm{CO}_{2}$. SGC-7901/Fu cells were cultured in identical medium containing $20 \mu \mathrm{g} / \mathrm{ml}$ 5-fluorouracil (5-FU) to maintain 5-FU drug resistance. For all experiments, cells were analysed at the logarithmic phase of growth.

Cell viability assays. SGC-7901/Fu cells were seeded in 96-well culture plates until the cells were at the logarithmic phase of growth. Each well received $20 \mu 1$ MTT (St. Louis, MO, USA) $(0.5 \mathrm{mg} / \mathrm{ml})$ and was incubated at $37^{\circ} \mathrm{C}$ for $4 \mathrm{~h}$. When the yellow solution appeared with blue crystals, the culture medium was removed, and $150 \mu \mathrm{l}$ of DMSO was added to each well to dissolve the crystals. The absorbance value (A490 nm) of each well was detected at $490 \mathrm{~nm}$ using a microplate reader. The experiment included time points at 24, 48, 72, 96 and $120 \mathrm{~h}$, and each group consisted of 8 replicate wells.

Cell transfection. Cells were seeded in 6-well culture plates, and transfection was performed when the cells reached $70 \%$ confluency. Transfection experiments included a control, $\mathrm{N}$-control, and RNAi treatment groups. Culture medium was replaced with serum-free RPMI-1640 culture medium containing both antibiotics. Cells were inoculated with $4 \mu \mathrm{l}$ of the appropriate virus solution (concentration of $1.5 \times 10^{9} \mathrm{Tu} / \mathrm{ml}$ ) for transfection in the presence of Polybrene (the recombinant RNAi lentiviral vector construct targeting the human $A B C C 4$ gene, LV-shRNA-ABCC4, was constructed previously in our laboratory). Infection was conducted for $12 \mathrm{~h}$, after which the culture medium was replaced with RPMI-1640 culture medium containing $10 \%$ FBS. The efficiency of RNA interference was determined by RT-PCR and western blotting.

RNA extraction and RT-PCR detection. Total RNA from the cells in each treatment group was extracted. The extraction procedure was conducted according to the manufacturer's instructions included with the RNAiso ${ }^{\mathrm{TM}}$ Plus kit (Takara,
Japan). After the RNA concentrations were determined, RT-PCR reactions were conducted using an RT-PCR reagent kit (Takara) according to the manufacturer's instructions. ABCC4, MDR 1 and $\beta$-actin primers were synthesised by Invitrogen. The primers for ABCC4 were as follows: upstream, 5'-CGCGTGTTCTTCTGGTGGCTC-3' and downstream, 5'-CTTTATCCCAGAACCCTTGC-3'. The primers for MDR1 were as follows: upstream, 5'-CCCATCATTGCAATAGC AGG-3' and downstream, 5'-GTTCAAACTTCTGCTCC TGA-3'. The primers for $\beta$-actin were as follows: upstream, 5'-CTGGGACGACATGGAGAAAA-3' and downstream, 5'-AAGGAAGGCTGGAAGAGTGC-3'. The PCR reaction contained $50 \mu \mathrm{l}$ in total volume, and the reaction conditions were as follows: $94^{\circ} \mathrm{C}$ for $2 \mathrm{~min}$ followed by 30 cycles of denaturation at $94^{\circ} \mathrm{C}$ for $30 \mathrm{sec}$, annealing at $61^{\circ} \mathrm{C}$ for $30 \mathrm{sec}$, and extension at $72^{\circ} \mathrm{C}$ for $30 \mathrm{sec}$. The PCR products were subjected to $1.2 \%$ agarose gel electrophoresis, and the results were scanned and analysed using a gel documentation system.

Detection of apoptosis. Cells from each treatment group were collected and prepared as a single-cell suspension. Staining for apoptotic cells was performed according to the instruction's manual included in the apoptosis reagent kit (Annexin V-FITC kit; BD Pharmingen, San Diego, CA, USA). Briefly, Annexin V and PI were added separately and stained at room temperature in the dark for $20 \mathrm{~min}$. Detection was performed using a flow cytometer (Becton-Dickinson, Franklin Lakes, NJ, USA).

Cell cycle analysis. Cells from each treatment group were collected and prepared as a single-cell suspension, after which the cells were fixed overnight in ice-cold $70 \%$ ethanol at $4^{\circ} \mathrm{C}$. Cells were stained with $1 \mathrm{ml}$ PI (containing $10 \mu \mathrm{g}$ RNase and $5 \mu 1$ Triton X-100) at $4^{\circ} \mathrm{C}$ in the dark for $30 \mathrm{~min}$. Cell cycle progression was analysed using a flow cytometer (Becton-Dickinson).

Detection of caspase activity. Cells from each treatment group were collected, and the activity of caspase-3, -8 , and -9 was detected according to the instruction's manual included with the caspase activity detection reagent kit (Beyotime, China). The analysis was performed using a fluorescence spectrophotometer at an excitation wavelength of $400 \mathrm{~nm}$ and an emission wavelength of $505 \mathrm{~nm}$.

Western blot analysis. After quantification using the bicinchoninic acid (BCA) method, the samples were loaded on an SDS-PAGE gel and separated. The proteins were transferred to a polyvinylidene fluoride (PVDF) membrane using the semi-dry method. The membrane was blocked overnight in $5 \%$ non-fat dry milk at $4^{\circ} \mathrm{C}$. After washing the membrane with TBST (Tris-buffered saline with Tween), the primary antibodies were added followed by a 1-h hybridisation at $37^{\circ} \mathrm{C}$ and TBST washes. Secondary antibodies were then added followed by a $1-\mathrm{h}$ hybridisation at $37^{\circ} \mathrm{C}$, a TBST wash, 5 min of the chromogenic reaction, and autoradiography. The optical density values were analysed and determined using the Quantity One software, and the results are expressed as the ratio of the sample optical density value to the optical density value of the internal reference. 
A
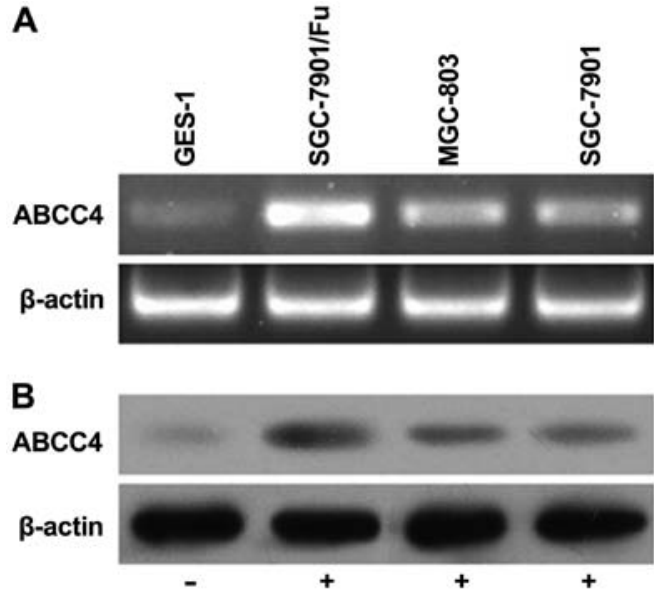

Figure 1. Expression of $A B C C 4$ in the GES-1, MGC-803, SGC-7901 and SGC-7901/Fu cells. (A) Analysis of ABCC4 gene expression in the 4 cell lines by RT-PCR. (B) Analysis of ABCC4 protein expression in the 4 cell lines by western blotting. Each independent experimental sample was repeated in triplicate.

Inoculation of nude mice. Our animal experiment protocol was approved by the Medical Ethics Committee of Guilin Medical University. A total of 20 male nude mice between 6 and 8 weeks of age at a body weight of $\sim 25 \mathrm{~g}$ were purchased from the Experimental Animal Centre of Guilin Medical University. Animals were randomly divided into 2 groups with 10 animals in each group. Cells $\left(5 \times 10^{5}\right)$ from the $\mathrm{N}$-control and RNAi groups were injected subcutaneously into each animal as appropriate. After 4 weeks of tumour growth, the mice were sacrificed by cervical dislocation, and tumour tissues were removed aseptically for further analysis.

Statistical analysis. Statistical analysis was performed using SPSS 16.0 software. All data are presented as the mean \pm standard deviation. Comparisons between groups were examined using one-way analysis of variance (ANOVA) and LSD-t. p-values $<0.05$ were considered statistically significant.

\section{Results}

ABCC4 gene expression is increased in multidrug-resistant gastric cancer $S G C-7901 / F u$ cells. RT-PCR and western blotting were used to detect the expression of $A B C C 4 \mathrm{mRNA}$ and protein in the GES-1, MGC-803, SGC-7901 and SGC-7901/Fu cells. Our results showed that the expression levels of $A B C C 4$ mRNA in the MGC-803, SGC-7901 and SGC-7901/Fu cells were higher than the $A B C C 4$ expression in the GES-1 cells. Notably, the $A B C C 4$ mRNA expression level was highest in the SGC-7901/Fu cells. In addition, western blotting results for ABCC4 protein expression levels in these 4 cell lines were consistent with the mRNA expression level results. These results revealed that the multidrug-resistant human gastric cancer SGC-7901/Fu cells expressed the highest levels of the ABCC4 transcript and protein (Fig. 1).

Knockdown of ABCC4 gene expression in multidrug-resistant gastric cancer $S G C-7901 / F$ u cells. RNAi was used to silence $A B C C 4$ gene expression in the SGC-7901/Fu cells. The cells were transfected with the recombinant RNAi lentiviral vector

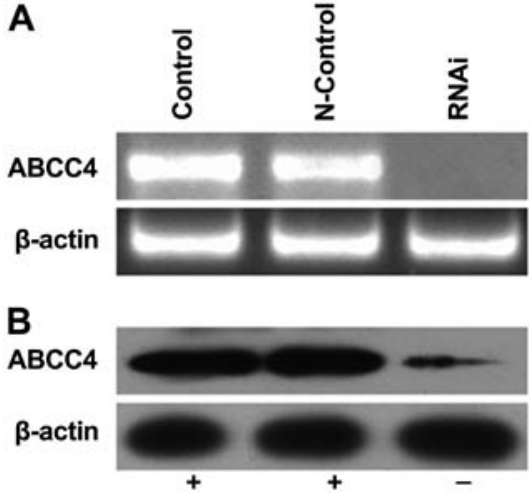

Figure 2. Silencing of $A B C C 4$ gene expression in the SGC-7901/Fu cells using RNA interference. (A) Detection of $A B C C 4$ gene expression levels upon $A B C C 4$ knockdown by RT-PCR. (B) Detection of ABCC4 protein expression levels upon $A B C C 4$ knockdown by western blotting. Each independent experimental sample was repeated in triplicate.
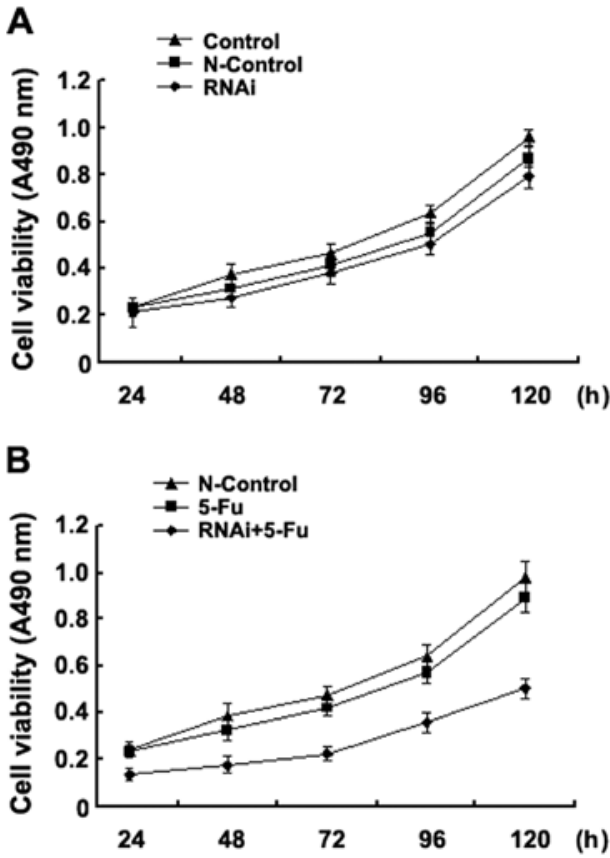

Figure 3. $A B C C 4$ gene knockdown enhances 5-FU-mediated inhibition of the proliferation of SGC-7901/Fu cells. (A) Relative to the control and N-control groups, cell proliferation in the ABCC4 RNAi group did not change significantly. (B) Relative to the $\mathrm{N}$-control and 5-FU groups, cell proliferation in the RNAi+5-FU group decreased significantly. Each independent experimental sample was repeated in triplicate.

targeting the human ABCC4 gene (LV-shRNA-ABCC4) or with a negative control construct, after which the expression levels of $A B C C 4 \mathrm{mRNA}$ and protein were analysed by RT-PCR and western blotting, respectively. Four days after transfection, the expression level of $A B C C 4$ mRNA was significantly downregulated relative to the control and $\mathrm{N}$-control groups. Consistently, the ABCC4 protein expression level was also decreased in the cells receiving the ABCC4 RNAi construct (Fig. 2).

Silencing of the ABCC4 gene sensitises SGC-7901/Fu cells to 5-FU. Next, we analysed the effect of the silencing 

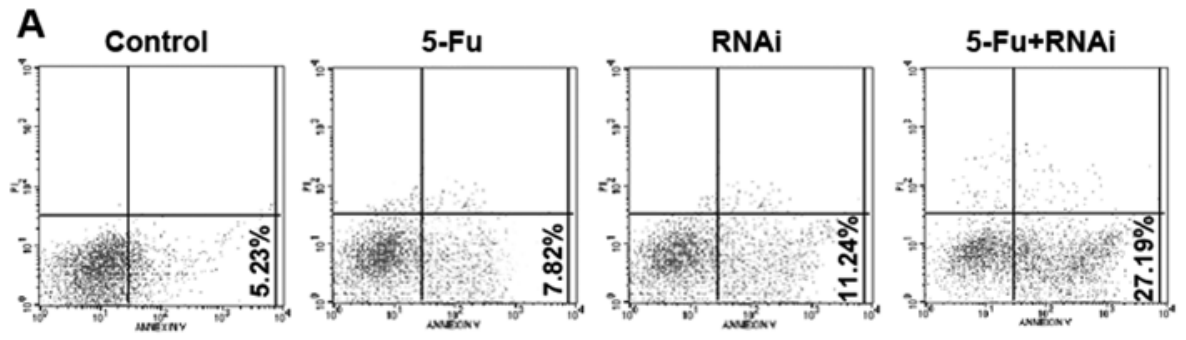

B
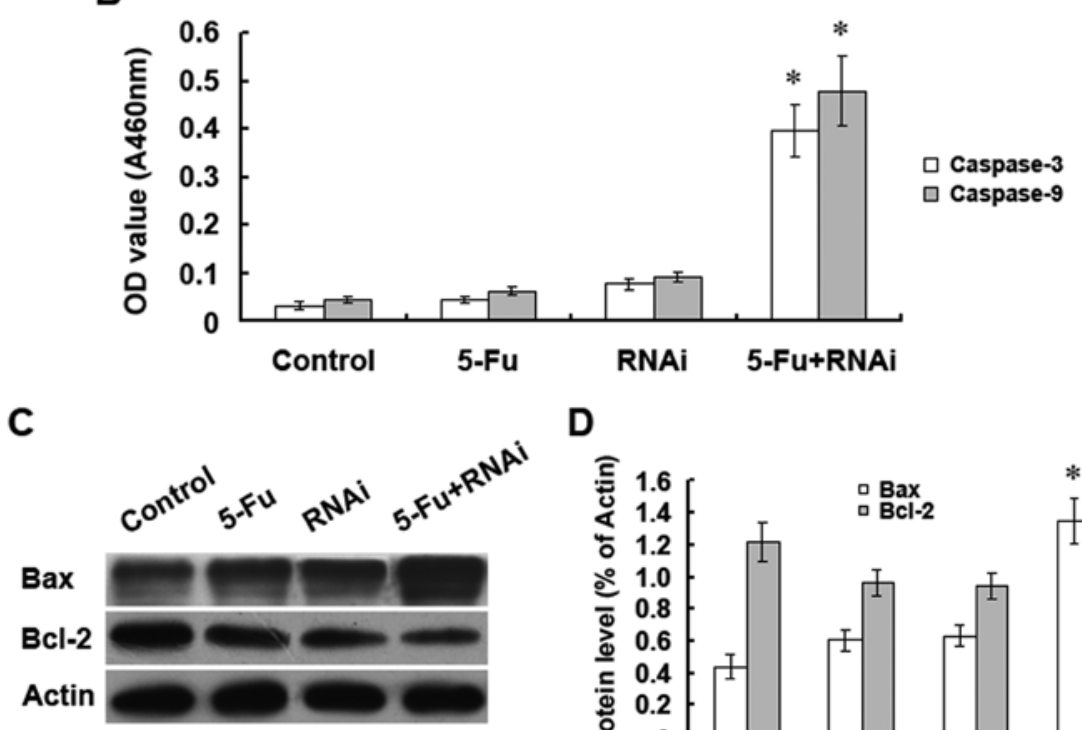

D

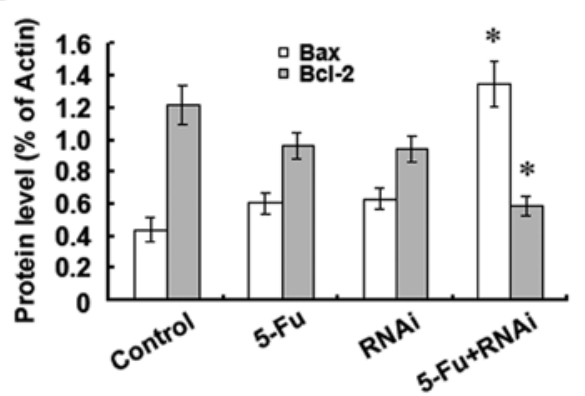

Figure 4. ABCC4 knockdown enhances 5-FU-induced apoptosis in SGC-7901/Fu cells. (A) Analysis of the apoptotic population in the SGC-7901/Fu cells upon $A B C C 4$ knockdown by flow cytometry. (B) Detection of caspase-3 and -9 activities upon $A B C C 4$ knockdown. (C and D) Analysis of Bax and Bcl-2 protein expression levels by western blotting after $A B C C 4$ knockdown. " $p<0.05$, each independent experimental sample was repeated in triplicate.

of the $A B C C 4$ gene on the proliferation capacity of the multidrug-resistant gastric cancer cell line SGC-7901/Fu. Our results showed that when compared with the control and $\mathrm{N}$-control groups, at all time points $(24,48,72,96$ and $120 \mathrm{~h}$ ) after transfection, the proliferation capacity of the SGC-7901/Fu cells did not change (Fig. 3A). However, upon treatment of the $A B C C 4$-silenced cells with a therapeutic dose of 5-FU, the proliferation capacity of the SGC-7901/Fu cells significantly decreased relative to the controls (Fig. 3B). Our results suggest that silencing of the $A B C C 4$ gene significantly enhanced the inhibition of SGC-7901/Fu cell proliferation by 5-FU.

Silencing of the ABCC4 gene enhances 5-FU-induced apoptosis in $S G C-7901 / F u$ cells. To investigate the molecular mechanisms underlying the increase in 5-FU-induced inhibition of SGC-7901/Fu cell proliferation upon $A B C C 4$ knockdown, we analysed cells for apoptosis by flow cytometry. Upon silencing of $A B C C 4$ expression in the presence of 5-FU, the apoptosis rate in the SGC-7901/Fu cells increased significantly relative to the other treatment groups (Fig. 4A). The expression levels of caspase-3 and -9 increased significantly in the $A B C C 4$-knockdown group (Fig. 4B). Furthermore, western blotting results showed that Bax protein expression was significantly increased, whereas Bcl-2 protein expression was decreased (Fig. 4C and D). These results suggest that silencing of $A B C C 4$ expression could enhance the ability of 5-FU to induce apoptosis in SGC-7901/Fu cells. Furthermore, the increase in apoptosis appears to be associated with the mitochondrial pathway.

ABCC4 knockdown sensitises SGC-7901/Fu cells to 5-FU-mediated cell cycle arrest. To further investigate the molecular mechanisms underlying the enhancement of 5-FU-induced inhibition of SGC-7901/Fu cell proliferation upon $A B C C 4$ knockdown, cell cycle analysis was conducted by flow cytometry. Our results showed that treatment with 5-FU or RNAi alone did not change the distribution of the cell cycle. However, ABCC4 knockdown in the presence of $5-\mathrm{FU}$ resulted in a significant increase in the cell population arrested in the G1 phase (Fig. 5A). In addition, the protein expression levels of the cell cycle proteins cyclin D1 and CDK4 were significantly decreased in the 5-FU+RNAi group (Fig. 5B and C). Since cyclin D1 and CDK4 proteins play indispensable roles in regulation of the G1 phase of the cell cycle, our results indicate that the enhancement of 5-FU-induced inhibition of SGC-7901/Fu cell proliferation upon $A B C C 4$ knockdown was achieved by downregulating cyclin D1 and CDK4 protein expression, thereby arresting the cell cycle in the G1 phase. 


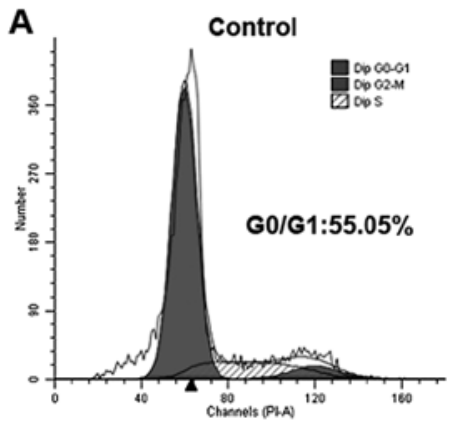

B

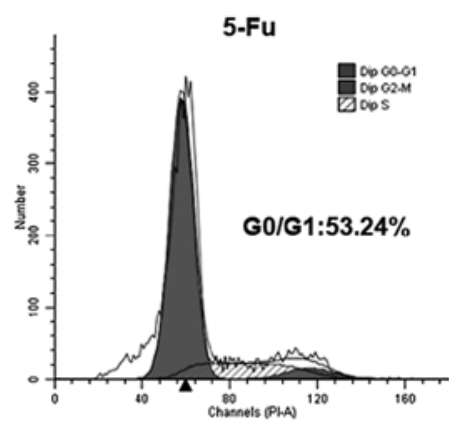

RNAi

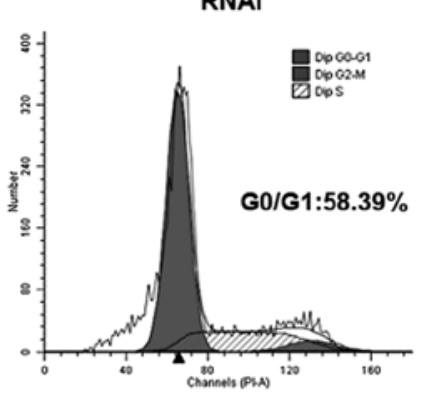

5-Fu+RNAi

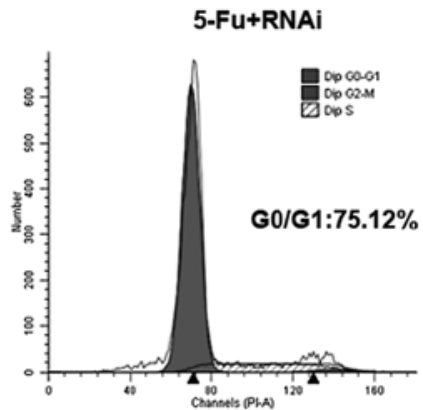

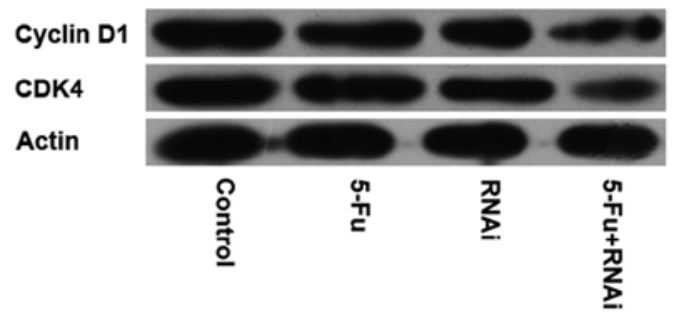

C

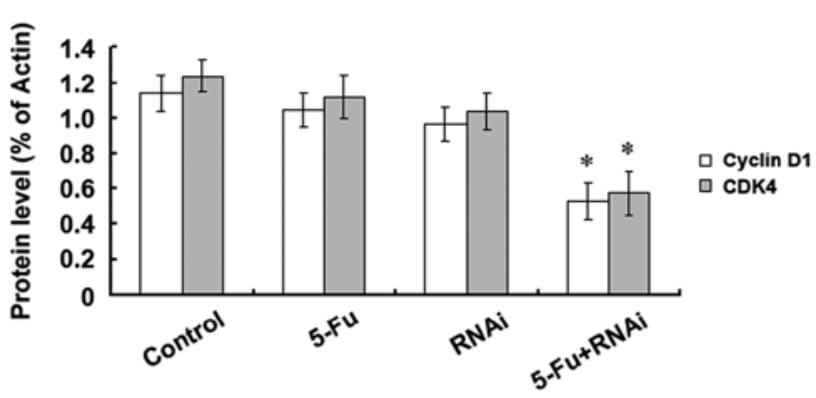

Figure 5. ABCC4 knockdown enhances 5-FU-induced cell cycle arrest in SGC-7901/Fu cells. (A) Cell cycle analysis upon $A B C C 4$ knockdown by flow cytometry. (B and C) Changes in the expression levels of the cell cycle proteins cyclin D1 and CDK4 upon $A B C C 4$ knockdown. "p $<0.05$, each independent experimental sample was repeated in triplicate.
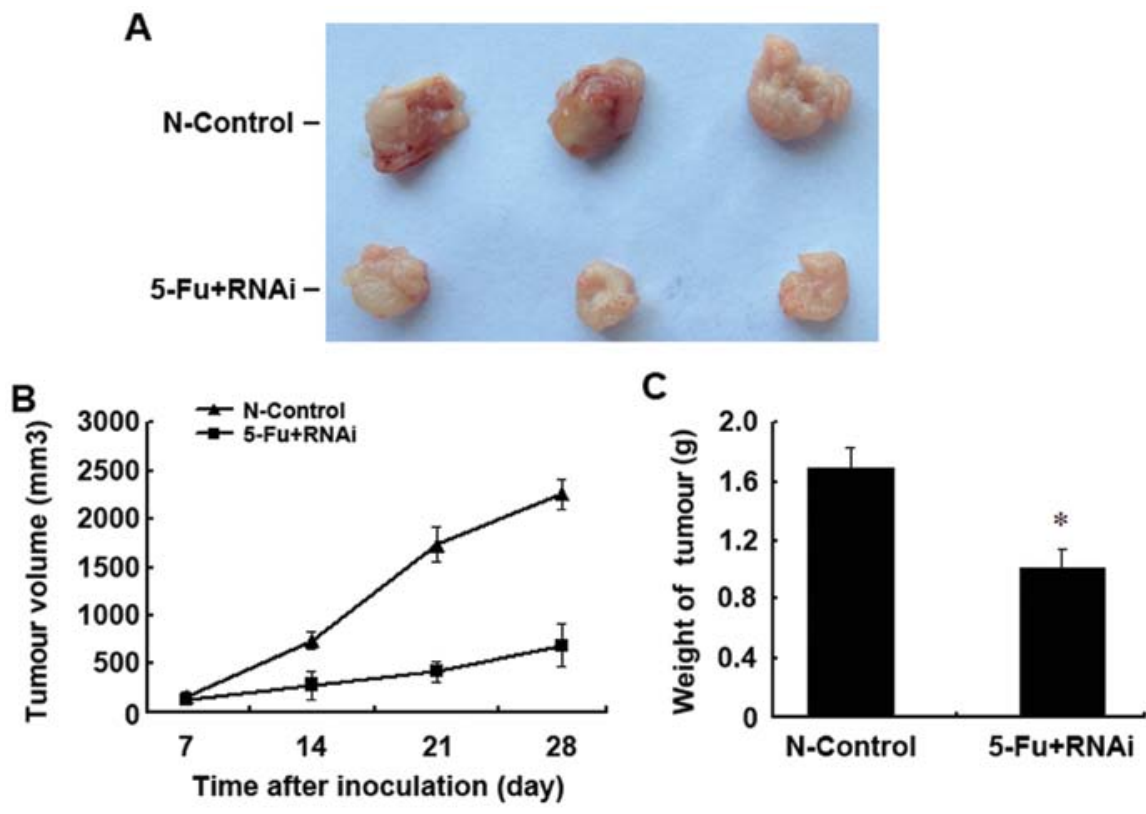

Figure 6. ABCC4 knockdown inhibits the growth of tumour xenografts in nude mice. (A) Surgical removal of tumour tissues on day 29 after implantation. (B) Tumour volumes were measured at different time points after implantation. (C) Tumour weights in the two groups on day 29 after implantation. * $p<0.05$, each independent experimental sample was repeated in triplicate.

ABCC4 knockdown inhibits the growth of tumour xenografts. The animal experiment program was approved by the Medical Ethics Committee of Guilin Medical University. The subcutaneous tumour xenograft model in the nude mice was conventionally established. Twenty nude mice were divided into equal treatment groups according to the xenograft implanted. One group received tumour implants that were subjected to $A B C C 4$ knockdown, whereas the other group received tumour implants that were subjected to non-specific control knockdown. Upon 5-FU treatment, we found that at all time points, the tumour volume in the 5-FU+RNAi group was significantly smaller than the tumour volume in the $\mathrm{N}$-control group. In addition, the weight of the surgically removed tumours in the 5-FU+RNAi group was also significantly lower than the tumour weight in the $\mathrm{N}$-control group (Fig. 6). Our results revealed that $A B C C 4$ knockdown in vivo significantly reversed the multidrug-resistance in human gastric cancer. 

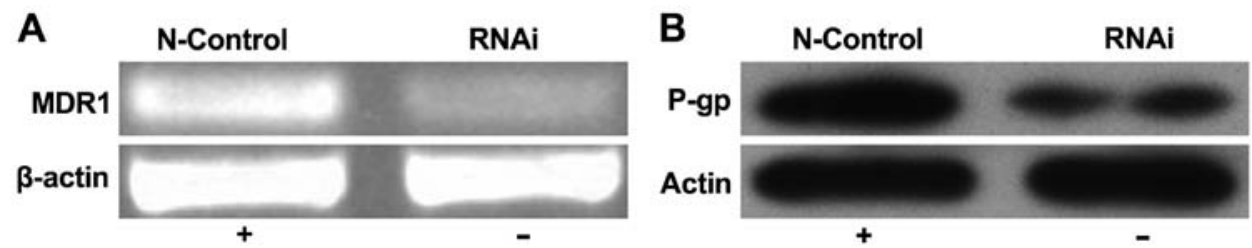

Figure 7. ABCC4 knockdown restores the sensitivity of SGC-7901/Fu cells to 5-FU through MDR1 downregulation. (A) MDR1 gene expression levels upon $A B C C 4$ knockdown by RT-PCR. (B) P-gp protein expression levels upon ABCC4 knockdown by western blotting. "p $<0.05$, each independent experimental sample was repeated in triplicate.

ABCC4 knockdown restores the sensitivity of $S G C-7901 / F u$ cells to 5-FU through MDR1. The generation of multidrug resistance is closely associated with the permeability glycoprotein (P-gp) transport protein (MDR1). To further investigate the mechanisms responsible for the increase in the sensitivity of the drug-resistant gastric cancer cell line SGC-7901/Fu to 5-FU upon $A B C C 4$ knockdown, the expression levels of the P-gp (MDR1) transport protein were analysed. Our results showed that upon $A B C C 4$ knockdown, MDR1 transcript and protein expression also decreased (Fig. 7). These results indicate that the observed increase in the sensitivity of SGC-7901/Fu cells to 5-FU treatment upon $A B C C 4$ knockdown could be associated with the concomitant downregulation of P-gp (MDR1) expression.

\section{Discussion}

ABC transport protein family 4 (ABCC4) is a member of the $\mathrm{ABC}$ transport protein family and is also known as multidrugresistance protein 4 (MRP4). ABCC4 was first identified on the basal membrane of prostate epithelial cells (3). The $A B C C 4$ locus is found on human chromosome 13q32, and the full length of the gene is approximately $312 \mathrm{~kb}$. ABCC4 contains 31 exons and encodes 1325 amino acids (13). ABCC4 lacks an N-terminal transmembrane domain and forms an MSD1-NBD1-MSD2-NBD2 structure. ABCC4 possesses the simplest structure of the proteins in the ABC family (14). ABCC4 can transport many endogenous and exogenous organic anions with various structures outside of the cell. This feature of ABCC4 provides cells with the ability to tolerate various cytotoxic compounds and to protect important tissues from xenobiotic damage (15). In addition, ABCC4 also affects drug metabolism in cells contributing to drug resistance.

The association between ABCC4 and cancer has become an area of interest for many researchers in recent years. Dong et al and Cai et al $(16,17)$ showed that ABCC4 was highly expressed in androgen-induced prostate cancer cells, and subsequent studies further confirmed the presence of a high copy number of ABCC4 mRNA in prostate cancer (18). The purpose of our study was to investigate the expression of $A B C C 4$ in drug-resistant human gastric cancer cells and to assess the potential for ABCC4-targeted inactivation in the treatment of drug-resistant gastric cancer. Our results showed that ABCC4 transcript and protein levels were increased in multiple human gastric cancer cell lines especially a drugresistant gastric cancer cell line. These results suggest that ABCC4 expression and the development of drug resistance in human gastric cancer are likely related hinting at the potential value of $\mathrm{ABCC} 4$ as a therapeutic target for drug-resistant gastric cancer.

To further elucidate whether ABCC4 is closely associated with the occurrence and development of drug resistance in human gastric cancer, we knocked down $A B C C 4$ expression levels in a drug-resistant human gastric cancer cell line and observed the biological changes. Our results showed that upon $A B C C 4$ knockdown, proliferation of the drug-resistant human gastric cancer cell line SGC-7901/Fu was inhibited. Furthermore, these cells displayed an increase in apoptosis and G1 cell cycle arrest. Knockdown of $A B C C 4$ also inhibited the growth of tumour xenografts in nude mice, suggesting that high expression levels of ABCC4 in drug-resistant human gastric cancer cells promote tumour cell proliferation. Previous studies have shown that high ABCC4 expression levels could promote the development of malignant tumours such as prostate cancer and ovarian cancer $(11,19,20)$. Consistently, our results showed that ABCC4 plays an important regulatory role in the proliferation of drug-resistant human gastric cancer cells.

To further investigate the role of ABCC4 in the regulation of the inhibition of drug-resistant human gastric cancer cell proliferation, we analysed apoptosis and cell cycle progression. The effect of ABCC4 expression on apoptosis has not been thoroughly researched. Our results showed that downregulation of $A B C C 4$ increased apoptosis in drug-resistant human gastric cancer cells. A study by Dai et al (21) showed that ABCC4 could regulate the mTOR signalling pathway, thereby regulating apoptosis. Consistently, our results showed that downregulation of $A B C C 4$ is closely associated with the inhibition of the proliferation of drug-resistant human gastric cancer cells and the occurrence of apoptosis. In addition, we also found that downregulation of $A B C C 4$ could arrest the cell cycle of drug-resistant human gastric cancer cells at the G0/G1 phase. These results collectively suggest that high ABCC4 expression levels observed in drug-resistant human gastric cancer cells are necessary for tumour cell proliferation. Furthermore, downregulation of $A B C C 4$ results in the inhibition of proliferation of drug-resistant human gastric cancer cells.

Several previous studies have shown that the mitochondrial pathway and death receptor pathway (22) are the classical apoptotic pathways in many tumour cells. Bcl-2 and Bax play important roles in the mitochondrial apoptosis pathway $(23,24)$. If Bax translocates from the cytoplasm to the mitochondrial membrane, permeability of the mitochondrial membrane changes to promote cytochrome $c$ release from the mitochondria into the cytoplasm thereby activating the caspase cascade culminating in apoptosis (25-27). In this study, we 
found that downregulation of $A B C C 4$ in drug-resistant human gastric cancer cells caused translocation of Bax and Bcl-2 and significantly increased caspase-3 and -9 expression levels. We speculated that during the mitochondrial pathway of apoptosis, release of cytochrome $c$ into the cytoplasm may activate the biological effects of caspase- 9 and -3 , thereby playing a key role in the activation of apoptosis. These results suggest that downregulation of $A B C C 4$ can induce apoptosis in drugresistant human gastric cancer cells through the mitochondrial pathway.

The occurrence of drug resistance in malignant tumours is thought to be closely associated with the abnormal expression of MDR1 and P-glycoprotein on the membrane of tumour cells. Therefore, some researchers have proposed that downregulation of these two proteins using a variety of methods could reduce the occurrence of drug resistance in tumour cells $(28,29)$. Abnormal expression of MDR1 and P-glycoprotein is usually closely associated with the generation of drug resistance in tumour cells. Studies have shown that downregulation of MDR1 and P-glycoprotein in drug-resistant tumour cells could inhibit the generation of drug resistance in tumour cells (30). The present study showed that downregulation of $A B C C 4$ in the presence of 5-FU significantly inhibited the proliferation and cell cycle progression of drug-resistant human gastric cancer cells and inhibited tumour cell growth in nude mice. In addition, the expression levels of MDR1 transcript and protein and P-glycoprotein on the membrane of tumour cells also decreased significantly. These results indicate that downregulation of $A B C C 4$ could alter the expression levels of MDR1 and P-glycoprotein on the cell membrane thus inhibiting proliferation of drug-resistant human gastric cancer cells.

In summary, the present study showed that ABCC4 is highly expressed in drug-resistant human gastric cancer cells. Furthermore, downregulation of $A B C C 4$ increased apoptosis and cell cycle arrest in drug-resistant human gastric cancer cells through the regulation of $\mathrm{Bcl}-2 / \mathrm{Bax}$ in the mitochondrial pathway, thereby restoring the sensitivity of the drug-resistant cancer cells to 5-FU. Our study may contribute to making a case for the targeting of ABCC4 for the clinical treatment of drug resistance in gastric cancer.

\section{Acknowledgements}

This study was funded by the Guangxi Zhuang Autonomous Region Health Department (Z2012403) and from Guangxi.

\section{References}

1. Parkin DM, Bray F, Ferlay J and Pisani P: Global cancer statistics, 2002. CA Cancer J Clin 55: 74-108, 2005.

2. Sun J, Song Y, Wang Z, Chen X, Gao P, Xu Y, Zhou B and Xu H: Clinical significance of palliative gastrectomy on the survival of patients with incurable advanced gastric cancer: a systematic review and meta-analysis. BMC Cancer 13: 577, 2013.

3. van de Ven R, de Groot J, Reurs AW, Wijnands PG van de Wetering K, Schuetz JD, de Gruijl TD, Scheper RJ and Scheffer GL: Unimpaired immune functions in the absence of Mrp4 (Abcc4). Immunol Lett 124: 81-87, 2009.

4. Russel FG, Koenderink JB and Masereeuw R: Multidrug resistance protein 4 (MRP4/ABCC4): a versatile efflux transporter for drugs and signalling molecules. Trends Pharmacol Sci 29: 200-207, 2008.
5. Keppler D: Multidrug resistance proteins (MRPs, ABCCs): importance for pathophysiology and drug therapy. Handb Exp Pharmacol 201: 299-323, 2011.

6. Norris MD, Smith J, Tanabe K, Tobin P, Flemming C, Scheffer GL, Wielinga P, Cohn SL, London WB, Marshall GM, Allen JD and Haber M: Expression of multidrug transporter MRP4/ABCC4 is a marker of poor prognosis in neuroblastoma and confers resistance to irinotecan in vitro. Mol Cancer Ther 4: 547-553, 2005.

7. Hendig D, Langmann T, Zarbock R, Schmitz G, Kleesiek K and Götting C: Characterization of the ATP-binding cassette transporter gene expression profile in Y79: a retinoblastoma cell line. Mol Cell Biochem 328: 85-92, 2009.

8. Heimerl S, Bosserhoff AK, Langmann T, Ecker J and Schmitz G: Mapping ATP-binding cassette transporter gene expression profiles in melanocytes and melanoma cells. Melanoma Res 17: 265-273, 2007.

9. Holla VR, Backlund MG, Yang P, Newman RA and DuBois RN: Regulation of prostaglandin transporters in colorectal neoplasia. Cancer Prev Res 1: 93-99, 2008.

10. Gillet JP, Efferth T, Steinbach D, Hamels J, de Longueville F, Bertholet V and Remacle J: Microarray-based detection of multidrug resistance in human tumor cells by expression profiling of ATP-binding cassette transporter genes. Cancer Res 64: 8987-8993, 2004.

11. Beretta GL, Benedetti V, Cossa G, Assaraf YG, Bram E, Gatti L, Corna E, Carenini N, Colangelo D, Howell SB, Zunino F and Perego P: Increased levels and defective glycosylation of MRPs in ovarian carcinoma cells resistant to oxaliplatin. Biochem Pharmacol 79: 1108-1117, 2010.

12. Gradilone A, Pulcinelli FM, Lotti LV, Trifirò E, Martino S, Gandini O, Gianni W, Frati L, Aglianò AM and Gazzaniga P: Celecoxib upregulates multidrug resistance proteins in colon cancer: lack of synergy with standard chemotherapy. Curr Cancer Drug Targets 8: 414-420, 2008.

13. Lamba JK, Adachi M, Sun D, Tammur J, Schuetz EG, Allikmets R and Schuetz JD: Nonsense mediated decay downregulates conserved alternatively spliced ABCC4 transcripts bearing nonsense codons. Hum Mol Genet 12: 99-109, 2003.

14. Ravna AW, Sylte I and Sager G: Binding site of ABC transporter homology models confirmed by ABCB1 crystal structure. Theor Biol Med Model 6: 20, 2009.

15. Adachi M, Sampath J, Lan LB, Sun D, Hargrove P, Flatley R, Tatum A, Edwards MZ, Wezeman M, Matherly L, Drake R and Schuetz J: Expression of MRP4 confers resistance to ganciclovir and compromises bystander cell killing. J Biol Chem 277: 38998-39004, 2002.

16. Dong Y, Zhang H, Gao AC, Marshall JR and Ip C: Androgen receptor signaling intensity is a key factor in determining the sensitivity of prostate cancer cells to selenium inhibition of growth and cancer-specific biomarkers. Mol Cancer Ther 4: 1047-1055, 2005.

17. Cai C, Omwancha J, Hsieh CL and Shemshedini L: Androgen induces expression of the multidrug resistance protein gene MRP4 in prostate cancer cells. Prostate Cancer Prostatic Dis 10: 39-45, 2007.

18. Ho LL, Kench JG, Handelsman DJ, Scheffer GL, Stricker PD, Grygiel JG, Sutherland RL, Henshall SM, Allen JD and Horvath LG: Androgen regulation of multidrug resistanceassociated protein 4 (MRP4/ABCC4) in prostate cancer. Prostate 68: 1421-1429, 2008.

19. Hagmann W, Jesnowski R, Faissner R, Guo C and Löhr JM: ATP-binding cassette $\mathrm{C}$ transporters in human pancreatic carcinoma cell lines. Upregulation in 5-fluorouracil-resistant cells. Pancreatology 9: 136-144, 2009.

20. Bagnoli M, Beretta GL, Gatti L, Pilotti S, Alberti P, Tarantino E, Barbareschi M, Canevari S, Mezzanzanica D and Perego P: Clinicopathological impact of ABCC1/MRP1 and ABCC4/ MRP4 in epithelial ovarian carcinoma. Biomed Res Int 2013: 143202,2013

21. Dai ZJ, Gao J, Kang HF, Ma YG, Ma XB, Lu WF, Lin S, Ma HB, Wang XJ and Wu WY: Targeted inhibition of mammalian target of rapamycin (mTOR) enhances radiosensitivity in pancreatic carcinoma cells. Drug Des Devel Ther 7: 149-159, 2013.

22. Nieminen AI, Partanen JI and Klefstrom J: c-Myc blazing a trail of death: coupling of the mitochondrial and death receptor apoptosis pathways by c-Myc. Cell Cycle 6: 2464-2472, 2007. 
23. Renault TT, Teijido O, Antonsson B, Dejean LM and Manon S: Regulation of Bax mitochondrial localization by Bcl-2 and $\mathrm{Bcl}-\mathrm{x}(\mathrm{L})$ : keep your friends close but your enemies closer. Int J Biochem Cell Biol 45: 64-67, 2013.

24. Lindsay J, Esposti MD and Gilmore AP: Bcl-2 proteins and mitochondria - specificity in membrane targeting for death. Biochim Biophys Acta 1813: 532-539, 2011.

25. Saito M, Korsmeyer SJ and Schlesinger PH: BAX-dependent transport of cytochrome c reconstituted in pure liposomes. Nat Cell Biol 2: 553-555, 2000.

26. Yuan S and Akey CW: Apoptosome structure, assembly, and procaspase activation. Structure 21: 501-515, 2013.

27. McIlwain DR, Berger T and Mak TW: Caspase functions in cell death and disease. Cold Spring Harb Perspect Biol 5: a008656, 2013.
28. Zhu CY, Lv YP, Yan DF and Gao FL: Knockdown of MDR1 increases the sensitivity to adriamycin in drug resistant gastric cancer cells. Asian Pac J Cancer Prev 14: 6757-6760, 2013.

29. Wang Y, Ma G, Wang Q, Wen M, Xu Y, He X, Zhang P, Wang Y, Yang T, Zhan P and Wei G: Involvement of CUL4A in regulation of multidrug resistance to P-gp substrate drugs in breast cancer cells. Molecules 19: 159-176, 2013.

30. Tang B, Du J, Wang J, Tan G, Gao Z, Wang Z and Wang L: Alpinetin suppresses proliferation of human hepatoma cells by the activation of MKK 7 and elevates sensitization to cis-diammined dichloridoplatium. Oncol Rep 27: 1090-1096, 2012. 